\title{
Automated Brunnstrom Assessment for Home Rehabilitation Based on GRNN Model
}

\author{
Ji-Ping Wang ${ }^{1}$, Li-Quan Guo ${ }^{1}$, Tian-Yu Sheng ${ }^{1,2}$, Da-Xi Xiong1 \\ ${ }^{1}$ Suzhou Institute of Biomedical Engineering and Technology Chinese Academy of Sciences, 215163 Suzhou, China \\ ${ }^{2}$ Shanghai University, 200444, Shanghai \\ ${ }^{1}$ wangjp@sibet.ac.cn
}

\begin{abstract}
To realize the upper extremity rehabilitation assessment for post-stroke intelligently, a new movement assessment model is established in this paper. A WBSN system consisted of two inertial sensors is employed to acquisit patients' rehabilitation data. The data is stored in both local and server database. An intelligent Brunnstrom assessment based on GRNN model is built on the server. In order to test the accuracy and reliability of the model, twenty patients and four physicians were chosen as volunteers to finish a standard rehabilitation action - touching shoulder with affected hand. The accuracy of the assessment model can be $93.6 \%$. The purpose to build an intelligent assessment is achieved. It makes patients train in the home setting and community possible.
\end{abstract}

\section{Introduction}

With the improvement of people's living standards, cerebrovascular diseases have become a major cause of people's death worldwide. Stroke is one of top three cause of death in them [1]. $70-85 \%$ of first-ever strokes are accompanied by hemiplegia [2]. According to the epidemiological statistics [3], over 1.6 million people died from stroke each year, about 157 per 100,000, in china and there are more than 2 million new stroke patients survived. It costs over 20 billion Chinese Yuan each year.

With the aggravating trend of aging population in china, hospital costs increase sharply. Therefore, during past decades, many researches focus on finding a method to improve rehabilitation effectiveness and reduce the costs. It is reported that home-based rehabilitation performs as well as hospital care [4]. Patients can practise under their personal training schedules with fewer constraint on time and location. However, patients cannot assess their training effect by themselves in the home setting without physician's help. It is difficult for the popular application of home-based rehabilitation [5]. Brunnstrom stage standard table is widely used in the clinical practice to assess rehabilitation stage for stoke patients' motor function [7-8]. Patients' motor function is classified into 6 stages by evaluating the accomplishment quality of standard actions listed in the table. It is difficult to be carried out in home settings and community because of its cost of time and need of physicians at hand [9]. An objective, quantitative and intelligent unsupervised assessment is required.

With the development of science and technology, wearable body sensor network systems (WBSNs) especially inertial sensors that include accelerometers, gyroscopes, and magnetometers - can assess the type, intensity, duration, frequency, and quality of various motion-related functional activities [9-10]. Patel et al. [11] have proposed a method based on Random. Forests algorithm to monitor rehabilitation outcomes in stroke patients with sensors attached to the hand, arm and trunk. 8 tasks were chosen from the Wolf Motor Function Test (WMFT) to estimate the total Functional Ability Scale (FAS) score. A wearable body sensor network system was developed and applied well in several hospitals and communities. However, the system could not provide an assessment of patients' motion function recovery.

Based on the works finished and the shortcomings of the system, a GRNN based motion function assessment model was proposed in this paper to assess the Brunnstrom stage for home-based rehabilitation.

\section{Methodolory}

\subsection{System}

The remote quantitative assessment system consists of three parts: the patient client, physician client, and web server. At the patient client side, patients will wear the sensors on the arm and wrist first and then finish the 
rehabilitation prescriptions which were made by the physicians in advance. During their training process, the wearable sensors will record the movement information in real time and wirelessly transmit the data to the computer and then upload to the web server database through Internet. Besides the storage function, another important function of web server is to analyse the movement data and give an assessment of Brunnstrom stage. According to the assessment, physicians can adjust the individualized rehabilitation prescriptions on time. The system can be used in the home setting and community anytime.

\subsection{Grnn Theory}

Generalized regression neural network (GRNN) is a variation to radial basis neural networks. It was suggested by D.F. Specht in 1991 [12]. It is similar to RBF neural network in structure. It consists of four parts (as shown in Figure 1): input layer, pattern layer summation layer and output layer (shown in Figure 1). Given a datasheet $X=[x 1$, $\mathrm{x} 2, \ldots, \mathrm{xn}] \mathrm{T}$, the corresponding target is $\mathrm{Y}=[\mathrm{y} 1, \mathrm{y} 2, \ldots$, yn]

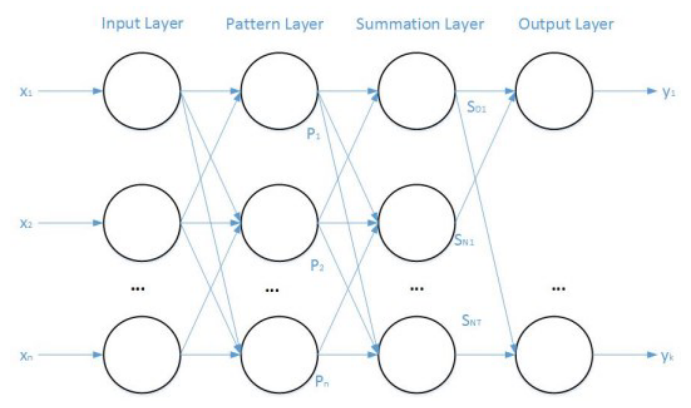

Figure 1 The structure of generalized regression neural network (GRNN)

The basic theory of GRNN is nonlinear regression analysis. Actually, the analysis of the dependent variable $\mathrm{Y}$ related to the independent variable $\mathrm{x}$ is to compute a maximum probability value $-\mathrm{y}$. the joint density function between $x$ and $y$ is supposed as $f(x, y)$, then the conditional mean can be descript as function (1):

$\dot{\hat{\mathrm{Y}}}=\mathrm{E}(\mathrm{y} / \mathrm{X})=\frac{\int_{-\infty}^{\infty} y f(X, y) d y}{\int_{-\infty}^{\infty} f(X, y) d y}$

Nonparametric estimation Parazen considered, sample datasheet $\left\{x_{i}, y_{i}\right\}_{i=1}^{n}$ supposed, the density function $\dot{\mathrm{f}}(\mathrm{X}, \mathrm{y})$ is that:

$\grave{\mathrm{f}}(\mathrm{X}, \mathrm{y})=\frac{1}{n(2 \pi)^{\frac{p+1}{2}} \sigma^{p+1}} \sum_{i=1}^{n} \exp \left[-\frac{\left(X-X_{i}\right)^{T}\left(X-X_{i}\right)}{2 \sigma^{2}}\right] \exp \left[-\frac{\left(X-Y_{i}\right)^{2}}{2 \sigma^{2}}\right]$

$\int_{-\infty}^{\infty} z e^{-z^{2}} d z=0$, the output $\widehat{Y}(\mathrm{X})$ is that:

$\widehat{\mathrm{Y}}(X)=\frac{\sum_{i=1}^{n} \mathrm{Y}_{i} \exp \left[-\frac{\left(X-X_{i}\right)^{T}\left(X-X_{i}\right)}{2 \sigma^{2}}\right]}{\sum_{i=1}^{n} \exp \left[-\frac{\left(X-X_{i}\right)^{T}\left(X-X_{i}\right)}{2 \sigma^{2}}\right]}$

$\widehat{Y}(X)$ is the weighted mean of the sample observations. While the smoothness factor $\sigma$ is very big, $\widehat{Y}(X)$ will be very close to the dependent mean of all samples. While the smoothness factor $\sigma$ approaches zero, $\widehat{Y}(X)$ will be close to the sample. In a word, when the factor $\sigma$ is an appropriate value, the dependent variable of all training samples is considered, the dependent variable of the point close to the predicted point will be given more weights.

\section{Experimental Configurations}

\subsection{Experimental Setup}

The experiment was carried out in the Rehabilitation Medical centre of the 2nd Hospital in Jiaxing, China. Twenty patients and four physicians were chosen as volunteers. The Brunnstrom stages of the patients were in the range II to $\mathrm{V}$ (estimated by the physicians). While patients in Brunnstrom stage VI perform no difference from the healthy people. The physicians served as the patients in Brunnstrom stage VI. All the patients attended in the test were listed in Table 1. There were eleven male patients and nine female patients, aged from 49 to 79 , involved in the experiment.

Table 1 the information of patients

\begin{tabular}{|c|c|c|c|c|}
\hline $\begin{array}{c}\text { Brunnstro } \\
\mathrm{m} \\
\text { Stage }\end{array}$ & case & $\begin{array}{c}\text { Gend } \\
\text { er } \\
\mathrm{M} / \mathrm{F}\end{array}$ & Age & $\begin{array}{c}\text { Paraplegia } \\
\text { side } \\
\text { left/right }\end{array}$ \\
\hline II & 2 & $0 / 2$ & $59.0 \pm 1.0$ & $2 / 0$ \\
\hline III & 8 & $4 / 4$ & $56.3 \pm 7.3$ & $4 / 4$ \\
\hline IV & 4 & $3 / 1$ & $\begin{array}{c}66.6 \pm \\
10.1\end{array}$ & $1 / 3$ \\
\hline $\mathrm{V}$ & 6 & $4 / 2$ & $65.6 \pm 7.5$ & $2 / 4$ \\
\hline
\end{tabular}

According to the Brunnstrom scale, the motion range of elbow, shoulder and forearm is a very important index to classify the stage of patient rehabilitation status. A standard motion, touching shoulder with affected hand, which can descript the index well was employed.

A WBSN system consisted of two inertial sensors was employed to collect patients' training data. The placements of sensors were shown in Figure 2, it is clear that the sensors were placed at the volunteer's forearm and the center of upper limb respectively with $\mathrm{x}$-axis of sensor parallel to the arm. The volunteers were required to finish the selected action five times and the action should be finished in $10 \mathrm{~s}$ with no pause. The data collected were stored in both local and server database. The assessment of the volunteers were given on server. The physicians, patients and their guardians have permission to check training data and the assessment on the web or mobile devices.

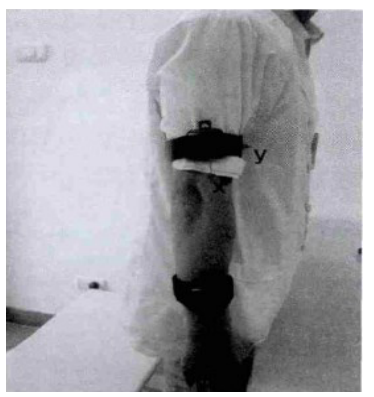

Figure 2 the sensors placed on arm 


\subsection{Feature Extraction}

The raw data was seen noisy due to sensor sample noise which reduced the data accuracy. A median filter was employed and works well in this paper. The raw data and denoised data were shown in Figure 3. It is observed that after filtering, the denoised data were smoother than raw data and the outliers caused by packet loss during transmission were removed.

Five types of feature were extracted (AMP, MEAN, RMS, JERK, and ApEn) during rehabilitation. Each inertial sensor has 3 axis and two sensors were employed, then there were total 30 features extracted. Besides, the energy, maximum of velocity, mean of velocity, std, entropy and maximum angle features were extracted too. These 42 features as shown in Table 2 were employed to assess the Brunnstrom stage of patients.
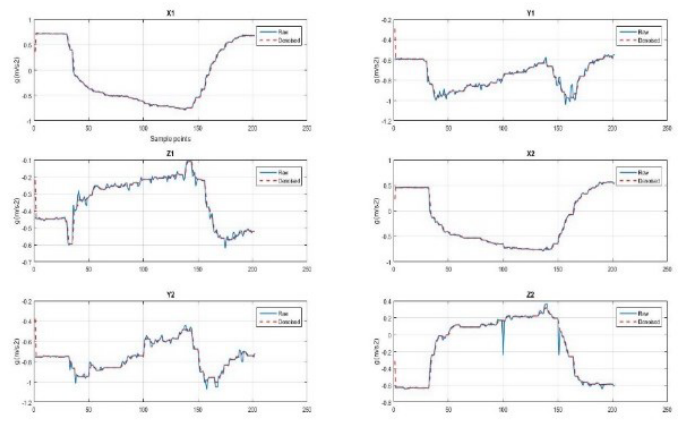

Figure 3 raw and denoised data of rehabilitation training

Table 2 features extracted from the rehabilitation data

\begin{tabular}{|c|c|}
\hline Feature & $\begin{array}{c}\text { Number of } \\
\text { features for } \\
\text { each } \\
\text { instance }\end{array}$ \\
\hline Maximum magnitude of data(AMP) & $2 \times 3$ \\
\hline Root mean square value of data(RMS) & $2 \times 3$ \\
\hline $\begin{array}{c}\text { Root mean square value of the } \\
\text { derivative of } \\
\text { data (JERK) }\end{array}$ & $2 \times 3$ \\
\hline Mean value of data( MEAN) & $2 \times 3$ \\
\hline Approximate entropy of data (ApEn) & $2 \times 3$ \\
\hline Energy (ENE) & 2 \\
\hline Maximum of the velocity(V max) & 2 \\
\hline Mean value of the velocity (V mean) & 2 \\
\hline STD of the velocity(V std) & 2 \\
\hline $\begin{array}{c}\text { Approximate entropy of the velocity (V } \\
\text { ApEn) }\end{array}$ & 2 \\
\hline Maximum angle (ANGLE max) & \\
\hline
\end{tabular}

\section{Results and Discussion}

\subsection{Results}

The predicted result of test sample is shown in Figure 4 . A sample in Brunnstrom stage III is predicted as stage IV; a sample in Brunnstrom stage IV is predicted as stage III; a sample in Brunnstrom stage $\mathrm{V}$ is predicted as stage IV. The predictive accuracy of samples is that the accuracy in stage II at $100 \%$, the accuracy in stage III at $93.8 \%$, the accuracy in stage IV at $90.0 \%$, the accuracy in stage $\mathrm{V}$ at $90.0 \%$, the accuracy in stage VI at $100 \%$. The study found that the errors might be cause by the factors listed below. Firstly, the lack of training samples used during the progress of building prediction model lead to an inaccurate model. Secondly, the patients performed well when their condition were comfortable and vice versa. In conclusion, the predictive accuracy of all test sample is $93.6 \%$. It means that the Brunnstrom assessment model based on GRNN has the extensive applicability.

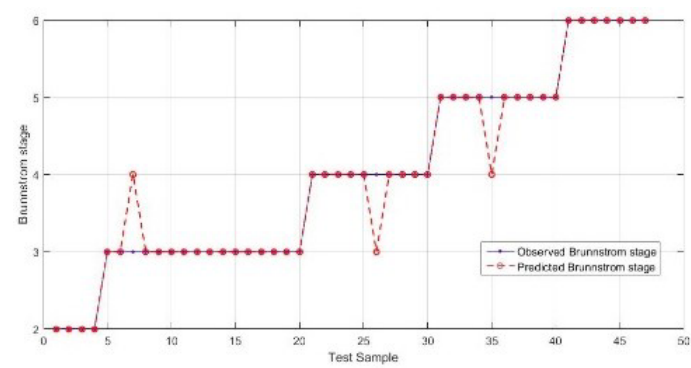

Figure 4 Observed results and predicted of the test samples

\subsection{Discussion}

An automated Brunnstrom assessment based on GRNN model is proposed to assess patients' motion function recovery in this paper. It can give the Brunnstrom stage of patients and help patients finish their assessment in home setting and community. An experiment was designed and carried in the rehabilitation center of 2 rd hospital in Jiaxing with the guidance of physicians. The test result shows that the model can assess patients' physical condition well and provides a reliable basis for making follow training plan. In future, more data will be collected to improve predictive model.

\section{References}

[1] M. L. Rao, China Guideline for Cerebrovascular Disease Prevention and Treatment. Beijing[M] People's Medical Publishing House, ch.1, (2007)

[2] Feigin, V.L, Forouzanfar, M.H, Krishnamurthi, etc. Global and regional burden of stroke during 1990 2010: Findings from the Global Burden of Disease Study 2010, The Lancet. 383, 245-255. (2014)

[3] Naghavi. M., Wang. H, Lozano. R, et al. Global, regional, and national age-sex specific all-cause and cause-specific mortality for 240 causes of death, 1990-2013: A systematic analysis for the Global Burden of Disease Study 2013.The Lancet, 385, 117 171(2015).

[4] L. Liu, D. Wang, K. L. Wong, and Y. Wang, Stroke and stroke care in china huge burden, significant workload, and a national priority, Stroke, vol. 42, no. 12, pp. 3651-3654, (2011).

[5] Langan J, DeLave K, Phillips L, et al. Home-Based Telerehabilitation Shows Improved Upper Limb Function in Adults with Chronic Stroke: A Pilot Study 
[J]. Journal of Rehabilitation Medicine, 45(2): 217220, (2013).

[6] Chen J, Jin, W, Zhang X.X, et al. Telerehabilitation Approaches for Stroke Patients: Systematic Review and Meta-analysis of Randomized Controlled Trials. [J]. Stroke Cerebrovascular Disease. 24, 2660$2668,(2015)$

[7] Nurdiana Nordin,Sheng Quan Xie and Burkhard Wünsche Assessment of movement quality in robot- assisted upper limb rehabilitation after stroke: a review, Journal of NeuroEngineering and Rehabilitation , 11:137(2014)

[8] Rachele Bertani, Corrado Melegari, Maria C. De Cola.etc. Effects of robot-assisted upper limb rehabilitation in stroke patients: a systematic review with meta-analysis, Neurological Sciences, pp:1-9 $\operatorname{May}(2017)$
[9] E.V. Olesh, S. Yakovenko, V. Gritsenko, Automated assessment of upper extremity movement impairment due to stroke [J]. PLOS ONE 9 (2014).

[10] Yu L, Wang Jp, Fang Q, et al. Brunnstrom stage automatic evaluation for stroke patients using extreme learning machine[C]. proceedings of the IEEE Biomedical Circuits and Systems Conference Proceedings, Hsinchu, Taiwan: 380-383, November (2012)

[11]S. Patel, R. Hughes, T. Hester, et al, A novel approach to monitor rehabilitation outcomes in stroke survivors using wearable technology, Proceedings of the IEEE, vol. 98, no. 3, pp. 450-461, (2010).

[12]D.F. Specht, A general regression neural network [J]. IEEE Transactions on Neural Net

[13]works, :568 - 576 ,Nov (1991) 